\title{
Escin may exert a synergistic anti-inflammatory effect with glucocorticoids
}

\author{
Lei-Ming Zhang, Tian Wang, Hua-Ying Fan, Xin Yu, Bing Han, Mei Zhu, Feng-Hua Fu
}

Department of Pharmacology, School of Pharmacy, Yantai University, Yantai, China; fufenghua@sohu.com

Received 16 October 2009; revised 15 December 2009; accepted 17 December 2009.

\begin{abstract}
Escin is a natural mixture of triterpenoid asponin isolated from the seed of the horse chestnut and demonstrates anti-oedematous and anti-inflammatory effects. As yet, the precise mechanisms by which escin exerts its antiinflammatory effects remain unclear. The data from current studies indicate that the anti-inflammatory properties of escin were attributed to its ability to reduce the adhesiveness of neutrophils and the associated release of inflammatory mediators; its ability to decrease histaminic and serotoninergic activities; its ability to inhibit phospholipase $A_{2}$; its ability to decrease nuclear factor-k $B$ activation and down-regulate the expression of tumor necrosis factor- $\alpha$. All these effects are similar to glucocorticoids. Moreover, escin depends on adrenal glands to exert its anti-inflammatory effects. Also, our recent research showed that the serum corticosterone level in mice did not increase after a 7-day intravenous injection of escin. The results support the hypothesis that escin may exert a synergistic anti-inflammatory effect with glucocorticoids. Confirming this hypothesis will play a role in elucidating the anti-inflammatory mechanisms of escin.
\end{abstract}

Keywords: Escin; Glucocorticoids; Inflammation; Synergism

\section{INTRODUCTION}

Escin, the major active principle from Aesculus hippocastanum (Hippocastanaceae), the horse chestnut tree, possesses diverse biochemical and pharmacological actions. It has been reported that escin has anti-oedematous, anti-inflammatory, and venotonic effects [1], and which currently has wide clinical use. Accumulated experi- mental evidence also suggests that escin exerts antioedematous and anti-inflammatory effects. Escin has been shown to be effective in preventing the formation of oedema in models of inflammation that reproduce the initial exudative phase, such as oedema induced in the paw by a series of irritative agents [2]. Additionally, escin shows a significant inhibition not only of the increase of capillary permeability induced by acetic acid, but also of the adhesion formation in animal model [3]. However, the anti-inflammatory mechanisms of escin are still unclear.

\section{PROOFS FOR THE HYPOTHESIS}

The studies showed that escin could attenuate brain injury, down-regulate the protein expressions of intercellular adhesion molecule (ICAM)-1 and E-selectin, and reduce the adhesiveness and migration of neutrophils [2, 4]. According to Matsuda [5], the anti-inflammatory effects of escin are mainly dependent on their anti-histaminic and antiserotoninergic activities. Another research [6] reported that escin dose-dependently prevented the hypoxia-induced activation of human endothelial cells, as evidenced by the inhibition of hypoxia-increased phospholipase A2, an enzyme responsible for the release of precursors of inflammatory mediators. In addition, escin can well alleviate the formation of inflammatory edema by blocking the increase in permeability through enhancing generation of prostaglandin F2 $\alpha$ [7]. Escin could significantly inhibit nuclear factor- $\kappa \mathrm{B}(\mathrm{NF}-\kappa \mathrm{B})$ activation and down-regulate the expression of tumor necrosis factor- $\alpha$ (TNF- $\alpha$ ), alleviating brain edema in traumatic brain injured rats [8].

The experiments listed above characterized that escin has potent anti-inflammatory effects and its anti-inflammatory mechanisms are similar to glucocorticoids (GCs) [9-11]. Additionally, an intriguing finding was that the anti-inflammatory effects of escin disappeared following adrenalectomy [12]. It suggests that the antiinflammatory effects of escin depend on GCs. However, our recent research showed the serum corticosterone level in mice did not increase after a 7-day intravenous 
injection of escin [13]. In another study, we further found escin to be a safe and potent anti-inflammatory drug with long effective anti-inflammation and without immunosuppression [14].

It is well known that GCs possess both anti-inflammatory and immunosuppressive effects. The anti-inflammatory and immunosuppressive effects of GCs rely on several molecular mechanisms, including direct effects on gene expression by the binding of glucocorticoid receptors (GR) to GC-responsive elements (i.e., the induction of annexin I and MAPK phosphatase 1), indirect effects on gene expression through the interactions of GR with other transcription factors (i.e., NF- $\kappa B$ and activator protein 1), and GR-mediated effects on second-messenger cascades (i.e., the PI3K-Akt-eNOS pathway) [15]. Unfortunately, because some of these mechanisms are also involved in physiologic signaling rather than inflammatory signaling, the therapeutic effects of GCs in inflammation are often accompanied by clinically significant side effects.

\section{THE HYPOTHESIS}

Based on the aforementioned data, we hypothesize that escin may exert a synergistic anti-inflammatory effect with GCs, which could explain the relationship described between escin and its anti-inflammatory mechanism, and the molecular mechanisms of the synergistic anti-inflammatory effect between escin and GCs may be derived from amplification of endogenous GC action through affecting GR or other elements in the signaling pathways. In fact, this hypothesis is not difficult to test. We can design experiments to confirm whether the combination of escin with GCs, which alone had no antinflammatory action in rodent animals by adrenalectomy, can greatly inhibit inflammation after the administration of physiological dose corticosterone. However, the difficulty in conducting these studies is how to precisely discover the molecular mechanisms of synergistic antiinflammatory effects between escin and GCs, that is, to determine escin how to affect the GC signaling pathway, from GR to other elements [15].

\section{CONSEQUENCES OF THE HPOTHESIS}

GCs are widely used to treat inflammatory diseases. However, GCs has multiple effects to inhibit the immune system and it is also associated with an increased susceptibility to infection and a risk for reactivation of latent tuberculosis. If our hypothesis could be proved correct, escin has its own virtue compared with GCs, as escin is not only a safe and potent anti-inflammatory $\mathrm{dr}$ ug, but also an anti-gastric ulcer agent [16]. Furthermore, it is easy to obtain and can be taken orally and venously with less side effects and complications. In conclusion, escin is a promising anti-inflammatory drug, promising wide clinical use within the population.

\section{ACKNOWLEDGEMENTS}

This study was supported by the National Natural Science Foundation of China (No.30772760), the 11th Five Years Key Programs for Science and Technology Development of China (No. 2008ZX09202-008), and Shandong Province Natural Science Foundation (No. Y2008C51).

\section{CONFLICT OF INTEREST STATEMENT}

All authors declare that there are no conflicts of interest.

\section{REFERENCES}

[1] Sirtori, C.R. (2001) Aescin: pharmacology, pharmacokinetics and therapeutic profile. Pharmacol Res, 44(3), 183-93.

[2] Guillaume, M., Padioleau, F. (1994) Veinotonic effect, vascular protection, antiinflammatory and free radical scavenging properties of horse chestnut extract. Arzneimittelforschung, 44(1), 25-35.

[3] Fu, F., Hou, Y., Jiang, W., Wang, R., Liu, K. (2005) Escin: inhibiting inflammation and promoting gastrointestinal transit to attenuate formation of postoperative adhesions. World J Surg, 29(12), 1614-20.

[4] Hu, X.M., Zhang, Y., Zeng, F.D. (2004) Effects of sodium-aescin on expression of adhesion molecules and migration of neutrophils after middle cerebral artery occlusion in rats. Acta Pharmacol Sin, 25(7), 869-75.

[5] Matsuda, H., Li, Y., Murakami, T., Ninomiya, K., Yamahara, J., Yoshikawa, M. (1997) Effects of escins Ia, Ib, IIa, and IIb from horse chestnut, the seeds of Aesculus hippocastanum L., on acute inflammation in animals. Biol Pharm Bull, 20(10), 1092-5.

[6] Arnould, T., Janssens, D., Michiels, C., Remacle, J. (1996) Effect of aescine on hypoxia-induced activation of human endothelial cells. Eur J Pharmacol, 315(2), 227-33.

[7] Longiave, D., Omini, C., Nicosia, S., Berti, F. (1978) The mode of action of aescin on isolated veins: relationship with PGF2 alpha. Pharmacol Res Commun, 10(2), 145-52.

[8] Xiao, G.M., Wei, J. (2005) Effects of $\beta$-Aescin on the expression of nuclear factor-kappa $\mathrm{B}$ and tumor necrosis factor-alpha after traumatic brain injury in rats. $J$ Zhejiang Univ SCI B, 6(1), 28-32.

[9] Auphan, N., Di-Donato J.A., Rosette, C., Helmberg, A., Karin, M. (1995) Immunosuppression by glucocorticoids: inhibition of NF-kappa B activity through induction of I kappa B synthesis. Science, 270(5234), 232-3.

[10] Hoeck, W.G., Ramesha, C.S., Chang, D.J., Fan, N., Heller, R.A. (1993) Cytoplasmic phospholipase A2 activity and gene expression are stimulated by tumor necrosis factor: 
dexamethasone blocks the induced synthesis. Proc Natl Acad Sci USA, 90(10), 4475-9.

[11] Cronstein, B.N., Kimmel, S.C., Levin, R.I., Martiniuk, F., Weissmann, G. (1992) A mechanism for the antiinflammatory effects of corticosteroids: the glucocorticoid receptor regulates leukocyte adhesion to endothelial cells and expression of endothelial-leukocyte adhesion molecule 1 and intercellular adhesion molecule 1. Proc Natl Acad Sci USA, 89(21), 9991-5.

[12] Hiai, S., Yokoyama, H., Oura, H. (1981) Effect of escin on adrenocorticotropin and corticosterone levels in rat plasma. Chem Pharm Bull, 29(2), 490-4.

[13] Zhang, L., Fu, F., Wang, T., Zhu, M. (2009) No up-regu- lation effects of escin on corticosterone in mice were observed. Basic Clin Pharmacol Toxicol, 105(Suppl. 1), 143-3.

[14] Wang, T., Fu, F., Zhang, L., Han, B., Zhu, M., Zhang, X. (2009) Effects of escin on acute inflammation and the immune system in mice. Pharmacol Rep, 61(4), 487-494.

[15] Rhen, T., Cidlowski, J.A. (2005) Antiinflammatory action of glucocorticoids - new mechanisms for old drugs. N Engl J Med, 353(16), 1711-23.

[16] Matsuda, H., Li, Y., Yoshikawa, M. (1999) Gastroprotections of escins Ia, Ib, IIa, and IIb on ethanol-induced gastric mucosal lesions in rats. Eur J Pharmacol, 373(1), 63-70. 